\title{
DEVELOPMENT OF A GROUP PSYCHOEDUCATION PROGRAM FOR PATIENTS WITH FIRST-EPISODE PSYCHOSIS
}

\author{
Á. De Diego G1, R. Mediavillla1,2, A. Muñoz S1, A. Orosa1, M.P. Vidal-Villegas¹, S. Cebolla1, E. Roman1 ${ }^{1}$, P. Sanchez Castro1,

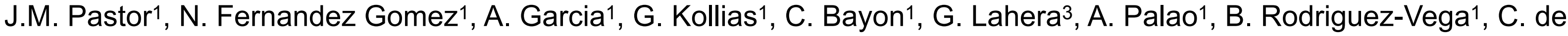 \\ Dios $^{1}$, L. Gotor ${ }^{1}$, R. Villanueva1 ${ }^{1}$, M.F. Bravo Ortiz¹.
}

${ }^{1}$ La Paz University Hospital, Psychiatry and Mental Health, Madrid, Spain

2National University of Distance Education UNED, Faculty of Psychology, Madrid, Spain

3University of Alcala- Faculty of Medicine, Psychiatry Department, Alcala de Henares, Spain.

\section{Objective}

To create a group psychoeducation (GPE) program for patients with first-episode psychosis (FEP)

\section{Background and aims}

Nowadays there has been an increasing interest on early identification and optimized treatment of FEP (1), whose programs are a priority in mental health $(2,3)$.

AgesMind is a randomized controlled trial which seeks to compare the efficacy and effectiveness of multicomponent GPE versus Mindfulness-based Social Cognition Training in people suffering from FEP (4).

The GPE program has been specifically developed in La Paz University Hospital within this context and is tailor-made for FEP patients in our recruitment area (Community of Madrid).

The aim is to explain the development of the afore-mentioned GPE program.

\section{Materials and methods}

We developed a GPE program for individuals with FEP. Inclusion to the study was allowed if the first onset of positive psychotic symtoms had taken place less than five years before.

We based on evidence supporting the use of $\operatorname{GPE}$ in $\operatorname{FEP}(5,6)$ as well as on our clinical practice experience. It has been developed by psychiatrists and psychotherapists with clinical experience in severe mental illness.

We fully developed a program that consists of 17 ninety minute long sessions throughout 9 months. It encompasses an intensive core of 8 weekly meetings, followed by 9 "booster sessions": 4 biweekly and 5 monthly sessions.

The program covers topics of therapeutic and prognostic interest. During the intensive sessions we discuss about possible causes of psychosis, potential diagnoses, and different therapeutic options, including drug treatment alternatives, their associated side effects, and psychosocial interventions.

Participants are also trained during the "booster" sessions to enhance their own welfare and relapse prevention plan. For this, they build on shared contents and information from the program's sessions.

All the sessions have the same structure, beginning with a review of possible incidents and the summary of the previous one.Then we go on to cover an specific interest topic. Participants are specifically encouraged to share their questions and suggestions regarding the program, including general aspects of the sessions and specific topics covered.

\section{Results}

We successfully developed a 17 session long GPE program. At the moment there are two on-going therapeutic groups in AgesMind's first and second randomizations.

Up until now sessions in GPE groups have been carried forward adequately and we have registered no adverse effects attributable to the therapy.

\section{Conclusions}

We think our GPE program is feasible and adequate to implement with FEP patients. We intend to implement it in future randomizations in order to further develop, evaluate, and be able to improve it.

\section{Distribution of intensive sessions}

\begin{tabular}{|l|}
\hline 1. Introduction and service information. \\
\hline 2. Understanding psychosis. \\
\hline 3. Phases model of psychosis. \\
\hline $\begin{array}{l}\text { 6. Psychoeducation about treatment II. Side effects of medical } \\
\text { treatment. }\end{array}$ \\
\hline $\begin{array}{l}\text { 7. Psychoeducation about treatment III. Long-acting injectable } \\
\text { medication. Discontinuation of medication. }\end{array}$ \\
\hline 8. Psychoeducation about treatment IV. Psychosocial interventions. \\
\hline
\end{tabular}

